\title{
About slow hydrostatic engine utilization from the structure of hydrostatic systems for machines and building equipments traction
}

\begin{abstract}
The relative comfortable solution of practical problems concerning the building and kinematics of different mechanisms through the use of hydrostatic driving confer them a large applicability in the structure of the machines and building equipments. The advantages of using hydrostatic driving in this area emphasized especially in the conditions of the actual rhythm of intensification and diversification of building works. In order to fulfil the needs of building equipments, the quantitative effort of their production must be supported by a continuous preoccupation concerning the obtaining of equipment with improved performances. The experience gained so far, the studies and the researches proved the machines and the building equipments with hydrostatic driving have an increased productivity, are more competitive and safer in exploitation.
\end{abstract}

Volume 7 Issue I - 202|

\author{
Daschievici Luiza, Ghelase Daniela \\ Department of Engineering Sciences and Management, \\ University of Galati, Romania
}

\section{Correspondence: Daschievici Luiza, Department of Engineering Sciences and Management, Dunarea de Jos, University of Galati, Romania, Email Luiza.Tomulesc@ugal.ro}

Received: January 18, 2021 | Published: February 08, 2021

Keywords: kinematics, building equipments, hydrostatic driven, hydrostatic traction

\section{Introduction}

In principle, the problem of the building equipments driving has two distinct appearances: working equipment driving and movement. Because the optimization of building equipments depends mostly on working equipment improvement, they took the advantages of hydrostatic driving utilization. Therefore, the majority of working equipments are currently hydrostatic driven.

As it concerns the traction, the situation is presented else different, in the sense that hydrostatic driving utilization still has numerous reserves. In present, classical or modernized mechanical driving systems are utilized for traction by using a hydrodynamic couple converter or improved gearboxes, the mixed traction consisting in the coupling of a hydrostatic quick engine to the simplified gearbox and still more recent, the complete hydrostatic systems tending to increase their preponderance. The first systems have the disadvantage of a low output, especially those with couple converter where the values of the output quickly diminish when operating in difficult regime, with great moments and small speed. Also, a lot of parts in relative movement causes many points of wear, what means an expensive care of. But, the complete hydrostatic traction offers at the most of advantages:

i. The relative simple transmission replacing the gearbox, the cardan drive, the differential and planetary axles with a hydraulic circuit consisting of static elements and only one moving element;

ii. Increased manoeuvrability by simplified controls and elements of automatic tunings utilization;

iii. easier maintenance;

iv. The possibility of extending the utilization of type elements;

v. Increased safety in exploitation.

These advantages are obvious especially for certain equipments, as chargers, roller blades, slashers, rolls compacters, where the working cycle includes frequent empty or charge movements, that is for the cases where the traction considerably influences the working performances of the equipment. In these situations, for the optimum performances, is to prefer the utilization of complete hydrostatic traction.

\section{Analysis of experimented data}

The main element of complete hydrostatic traction is the hydrostatic slow engine. The engine transforms the hydrostatic energy of working fluid into small speed rotating movement (below 300 rot. min.). Working at small speed, the hydrostatic slow engine can be coupled directly to the driving wheels of driving mechanism. Utilized as an ultimate element of the running system, a hydrostatic slow engine must satisfy the requirements requested by the equipment working conditions: developing an active moment, big enough when charge is present and the proper speed when there is no charge. Therefore, the working regime of a hydrostatic slow engine used for traction is framed by the extreme two points: maximum moment - minimum speed and minimum moment - maximum speed.

The used power depending on the wheel active moment and on the revolution, from the condition that the used power must not exceed the value of the source power, which is considered constant, results parabolic dependency between the two parameters: wheel active moment and the hydrostatic speed. So, for the equipments with hydrostatic slow engines, a traction characteristic as a parabolic functions is obtained.

The wheel active moment depending on the specific volume of hydrostatic engine and on the working pressure, and the speed depending on the source flow, if the specific volume is considered as a constant parameter of hydrostatic engine, in the same conditions of having a constant value of the used power, results a parabolic interdependence between the flow and the working pressure (Figure 1) (Figure 2).

Because the working pressure depending on driving wheel resistance can not be checked up, the parabolic interdependence of the two parameters is possible only by flow modification. This is achieved through the utilization as the power source of variable flow and automatic power adjustment pumps which use as an input signal the value of the pressure, very frequent used-up system in hydrostatic 
driving. Therefore, the extreme values of the pressure parameters are limited by the hydrostatic engine and power source parameters: the maximum pressure maxim and the maximum flow.

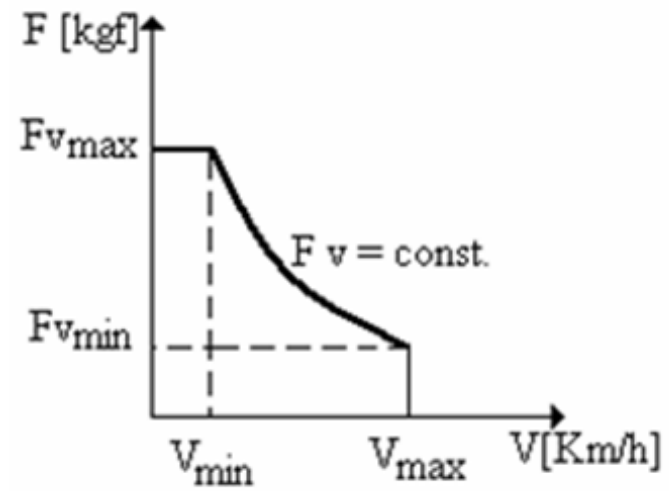

Figure I volume of hydrostatic engine and on the working pressure.

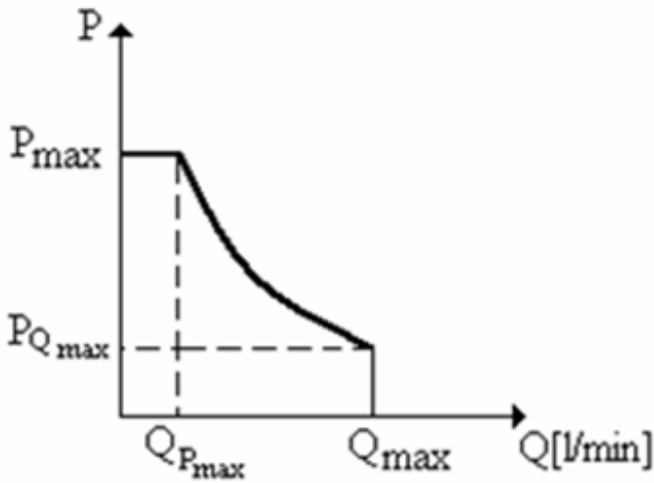

Figure 2 Results a parabolic interdependence between the flow and the working pressure.

It was previously mentioned that the specific volume is a constant parameter of hydrostatic slow engines, what corresponds the reality. With the exception of some cases of using the hydrostatic slow engines with specific variable volume, they are executed in the variant with specific constant volume, having one or many regimes of operation obtained through the step-by-step change of the specific volume.

The utilization of hydrostatic slow engines with more than one regime of operation considerably improves the traction characteristic. For instance, the using a hydrostatic slow engine with two regimes - one of them having a specific volume $\mathrm{n}$ times higher than the second - for the same characteristic of power adjustment, a traction characteristic $\mathrm{n}$ times higher is obtained compared to the same characteristic corresponding to single working regime of hydrostatic engine. Taking into account the output signal transmitting, two variants of hydrostatic slow engine can be distinguished: with revolving shell and with revolving axle. The first variant is better for equipments traction because of the possibility of embedding in the driving wheels rim, therefore is frequently used for equipments traction. The hydraulic installations used for the complete hydrostatic traction of the building equipments can be in open shape, half-closed and closed. The advantages of the second and third type against the first one, consist in:

1. Superior output due to the absence of hydraulic loss in the distribution block;

2. More economical construction of the hydraulic installation and reservoir.

Globally, the production of hydrostatic slow engines is increasing, numerous firms including them in their programs of manufacturing in different constructive variants and typo dimensional ranges, slow, middle and hard (according to nominal moments with values between tenths and thousands of daNm). Many of them are especially manufactured to be used for the complete hydrostatic traction of the equipments. Thus, were produced hydrostatic slow engines with radial pistons and fixed shell (central revolving axle) for nominal moments between 40 and $4000 \mathrm{daNm}$, pressures of $300 \mathrm{kgf} \mathrm{cmp}$, with one, two or three speeds ranges achieved through the radial pistons distribution shut

Because this type of hydrostatic engines the bearing element of the output signal is the central axle, their using for the equipments traction is relative heavy because of their attaching on the frame and coupling to the driving wheel. Firm SISU produces hydraulic slow engines with mobile shell which can be inserted in the wheel rim, so they are used for equipments traction. Because this type of hydrostatic engines the bearing element of the output signal is the central axle, their using for the equipments traction is relative heavy because of their attaching on the frame and coupling to the driving wheel. Firm SISU produces hydraulic slow engines with mobile shell which can be inserted in the wheel rim, so they are used for equipments traction. The engines produced by this firm are placed on fixed or oscillating decks or on railway axles. The implementation of complete hydrostatic traction for equipments construction is still in the progress.

Our preoccupations in this sense are conditioned by the researches and the experimentations performed in the sight of the assimilation in the serial manufacturing of hydrostatic slow engines. We experimented two sizes of hydraulic slow engine with radial pistons - for easy and medium working regimes - with revolving shell and with parameters shown in Table 1 were experimented.

Table I For easy and medium working regimes - with revolving shell and with parameters

\begin{tabular}{llllll}
\hline \multirow{2}{*}{ Parameter } & \multicolumn{3}{l}{ Values } \\
\cline { 2 - 6 } & U.M. & Hydr.engine I & Hydr.engine 2 \\
\cline { 2 - 6 } & & Regime I & Regime II & Regime III & Regime IV \\
\hline Maximum moment & $\mathrm{daNm}$ & 250 & 250 & 750 & 750 \\
Specific volume & $\mathrm{l} / \mathrm{rot}$ & 0,7 & 0,8 & 1,78 & 0,517 \\
Working speed & $\mathrm{rot} / \mathrm{min}$ & $0-100$ & $0-300$ & $0-80$ & $0-250$ \\
Radial pressure & $\mathrm{daN}$ & 3000 & 3000 & 10000 & 10000 \\
\hline
\end{tabular}




\section{Conclusion}

The original solutions of solving the problems, applied to these engines constituted the our objective. Among these, the most important refers to the achieving way of the two working regimes. In the paper we present the recent engines experimented where the radial pistons were replaced through sets of two pistons concentrically inserted having the active surfaces corresponding to the working regimes achieved through the commutation of the distribution from a piston to another. The advantage is that by using this solutions, a smaller size was obtained compared to the one of other hydrostatic slow engines with the same nominal moment.

The researches and the experimentations performed with the two hydrostatic slow engines emphasized the advantages of their utilization to the building equipment's traction. Thus, using the hydrostatic slow engine to the frontal charger with direction through side-slip of $45 \mathrm{CP}$, it was obtained an experimental model with an increased manoeuvrability and a higher output.

The same advantages were obtained using hydrostatic slow engines type 2 to a frontal charger with direction through side-slip of $60 \mathrm{CP}$ and a modified variant to the obtaining of an experimental model of frontal charger of $120 \mathrm{CP}$ with twin frame. The report of $1 / 3$ of the specific volumes of the two working regimes of hydrostatic slow engines proved to be enough for obtaining an acceptable traction, comparable to similar types of chargers.

Thus, for the experimental model of charger of $120 \mathrm{CP}$, choosing the elements of the driving system a traction characteristic limited by value of $V \max =35,2 \mathrm{~km} / \mathrm{h}$ and a maximum wheel force of $6500 \mathrm{daN}$ were obtained (Figure 3).

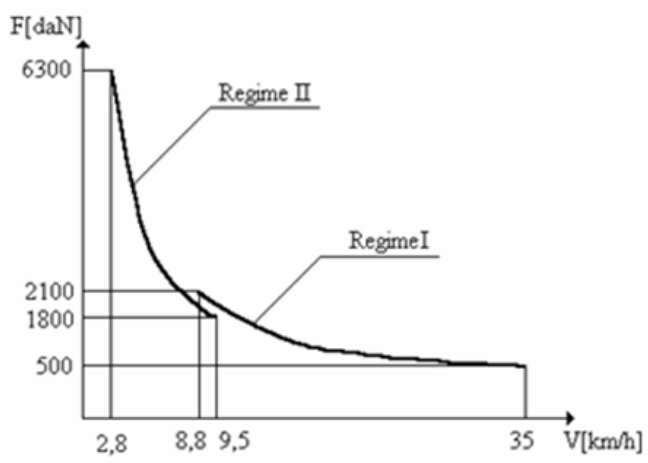

Figure 3 Maximum wheel force of $6500 \mathrm{daN}$ were obtained.
Following the curve, it was stated that a single control (corresponding to passing from a regime to another) is enough in order to attain any value of the parameters. In the hypothesis that the same charger would have had mechanic traction, for the same values of the parameters a gearbox with four steps would have been necessary, therefore three controls for maximum values of traction parameters. The hydrostatic slow engines are completed with a shoe-brake, figured in same shell, resulting thus a compact building which can be inserted as free unit in a wheel rim. This quality, completed by their fixation on the frame and joining to the feeder installations are relative simple, permits their using to the traction of different equipments. Using proper backing plates, the hydrostatic engines can be mounted directly on frame, or on fixed, oscillatory or directional decks, thus permits that through simple accommodations and modifications to the project, these to be applied to the following equipments: hydraulic excavators, roller blades, rolls compacters. ${ }^{1-6}$

\section{Acknowledgements}

None.

\section{Conflicts of interest}

The author declares that there was no conflict of interest.

\section{References}

1. Mihailescu St. s.a. - Masini de constructii, Ed. Tehnica, Bucuresti, 1985.

2. Rexroth-worldwide hydraulics, hydraulics \& pneumatics, March. 1987.

3. Prospectuses and catalogs of companies: Abex D (S.U.A.), Athos, B (Germany), Svendborg (Denmark), etc.

4. https://en.wikipedia.org/wiki/Sisu_M-161\#Engine

5. Dumitrache I, Catană I, Militaru A. Fuzzy controller for hydraulic servo systems. ifac international workshop on trends in hydraulic \& pneumatic components \& systems, Chicago, Illinois. 1994.

6. Magazine collections: Hydraulics and pneumatics, energie fluide et lubrification, olhydraulik and pneumatik, hydraulics and pneumatics, mechanical power. etc. 